\title{
Changes in the Structure of the Benzylpenicillin Sodium Salt Molecule under the Pulsed Magnetic Field
}

\author{
Natalia A. Rodenko ${ }^{1,2^{*}}$, Valentina A. Zhukova ${ }^{1}$, Tatyana I. Vasilyeva ${ }^{1}$, \\ Vladimir A. Glushchenkov ${ }^{1,2}$, and Irina A. Belyaeva ${ }^{1,2}$ \\ ${ }^{1}$ Samara National Research University, 34 Moskovskoe shosse, Samara 443086, Russia \\ ${ }^{2}$ Samara Federal Research Centre of the Russian Academy of Sciences, 3A Studencheskiy pereulok, Samara 443001, \\ Russia
}

*e-mail: t.rodenko@mail.ru

\begin{abstract}
The study aims at the Fourier spectra of an antibiotic exposed to a high-intensity pulsed magnetic field. The applied frequency was $\mathrm{f}=40 \mathrm{kHz}$, with a number of pulses $\mathrm{n}=1$, at intensities of the pulsed magnetic field $\mathrm{H}=0.09 \times 10^{6} \mathrm{~A} / \mathrm{m}, \mathrm{H}=0.50 \times 10^{6} \mathrm{~A} / \mathrm{m}, \mathrm{H}=0.82 \times 10^{6} \mathrm{~A} / \mathrm{m}$. It was assumed that under the conditions of the pulsed magnetic field there might be a change in the structure of the drug molecule. The research has revealed that an increase in the pulsed magnetic field power leads first to a decrease in the intensity of the band in the Fourier spectrum, and then to its increase. In addition, changes were recorded in the amide group of benzylpenicillin sodium salt molecules depending on the intensity of the pulsed magnetic field applied to the antibiotic. (C) 2021 Journal of Biomedical Photonics \& Engineering.
\end{abstract}

Keywords: antibiotic; Fourier spectroscopy; magnetic field intensity; biological activity.

Paper \#3387 received 15 Dec 2020; revised manuscript received 31 Jan 2021; accepted for publication 18 Mar 2021; published online 31 Mar 2021. doi: 10.18287/JBPE21.07.010305.

\section{Introduction}

Currently, the question of the effectiveness of antibiotics has become a matter of great urgency. As a rule, antibiotics are used in the form of solutions, which results in a complex water-antibiotic-biosystem. Here, the problem of the interaction of a water molecule with a solid (powdered) antibiotic comes to the fore. In Ref. [1], it was noted that antibiotics in the form of solid samples (powders) are stable during storage, while those in the form of aqueous solutions quickly lose their biological activity. Therefore, it is important to find out the reason for the increase in the antibacterial activity of the benzylpenicillin sodium salt after being exposed to the pulsed magnetic field (PMF). The structure of a benzylpenicillin molecule (Fig. 1) demonstrates such characteristic features of penicillin antibiotics as the presence of a $\beta$-lactam ring, an amide group, and a thiazole cycle.

In Ref. [2], the potential of benzylpenicillin was calculated as an index of the reactivity of the antibiotic. It was shown that during the transition from a neutral penicillin molecule to its anion, the dipole moment increases from 4.7 D to $18.2 \mathrm{D}$, and the benzylpenicillin anion molecule takes on a complex semispherical spatial structure. The authors showed that in this case, the carbonyl groups are located on the outer side.

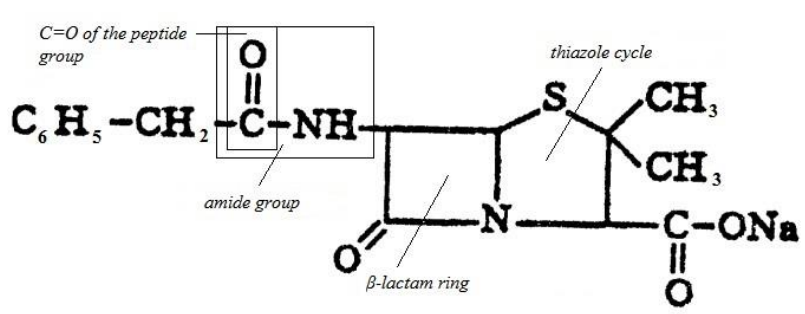

Fig. 1 Structural formula of benzylpenicillin sodium salt.

The authors of study [3] used quantum-chemical calculations to study the interaction of solid samples of benzylpenicillin with water and determined the location of water molecules during the formation of hydrate structures of the antibiotic. In this case, a threedimensional grid of hydrogen bonds is formed, which includes all the functional groups of the antibiotic. 
Jason Potticary et. al. [4] crystallized coronene (polyaromatic hydrocarbon) into another polymorph using the method of growing crystals under the conditions of the magnetic field action. Crystals, grown in a magnetic field with an induction of $1 \mathrm{~T}$, differ in their appearance from crystals grown in a zero field, and retain their structure under environmental conditions. The researchers found the new form to be the most stable at low temperatures. As a result of the new supramolecular configuration, significant changes in the electronic, optical and mechanical properties were noted.

In another similar work, polymorphism during the crystallization of drugs by a magnetic field was investigated (Project №736899, UK, the Netherlands, Ireland). The European researchers have already established that it is possible to synthesize specific polymorphs of pharmaceutical substances, such as carbamazepine, indomethacin, and paracetamol. They found out [5] that concretive crystalline forms could exhibit unique properties that may not be shown by other forms of the same substance.

The researchers of the Kalinin Medical Institute [6] made an attempt to trace the action of the constant magnetic field on barbamil (sleeping substance). Three times, at different times of the year, the experimental batch of mice received a sleeping substance that had been previously exposed to a constant magnetic field. It was observed that under the influence of the "magnetized" sleeping pill the mice slept longer than the animals from the control group that received the same dose of the "nonmagnetized" drug.

Thus, the effect of drugs on the organism may depend on the characteristics of drugs: physical properties, chemical structure, dose (concentration), as well as on the medicinal form of the drug. The influence of physical factors on the drug can enhance its biological effect in the organism [7].

In our previous work [8], we found a $12-24 \%$ increase in the antibacterial effect of benzylpenicillin sodium salt processed by a pulsed magnetic field with the field intensity ranging from $0.09 \times 10^{6} \mathrm{~A} / \mathrm{m}$ to $1.23 \times 10^{6} \mathrm{~A} / \mathrm{m}$ at the frequency $\mathrm{f}=40 \mathrm{kHz}$ (single-turn inductor) and a number of pulses $n=1$. The pulsed magnetic field action was carried out on a powdered pharmaceutical preparation. Fig. 2 presents the scheme of the PMF action on the drug: inductor 1, pulse current generator 2, PMF sensor 3, and oscilloscope 4. The PMF sensor is connected to the oscilloscope 4 . A vial 5 filled with about $4 \mathrm{~mm}$ of benzylpenicillin is placed in the middle of inductor 1 , after which the pulsed magnetic field treatment is performed.

To determine sensitivity of the bacterium Escherichia coli (E. coli.) to benzylpenicillin, antibacterial discs were used. The standard disc-diffusion method with sterile dilutions was developed in late 1960s to early 1970s [9] and since then has not undergone any significant changes from a methodological point of view. This method can be used for individual antibacterial therapy to treat the concretive infection in patients, to monitor the spread of antibiotic resistance in a certain area, as well as to study new chemical compounds for the presence of antibacterial activity [10].

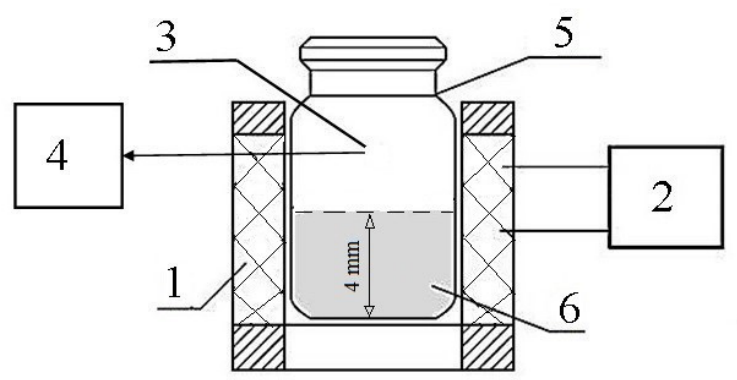

Fig. 2 Scheme of the pulsed magnetic field action on benzylpenicillin.

The study [11] of benzylpenicillin sodium salt in the powdered form $(1 \mathrm{~g})$ revealed that after the exposure of benzylpenicillin sodium salt to the pulsed magnetic field, the antibiotic obtained the concentration of $0.01 \mathrm{~g} / \mathrm{ml}$. The action of benzylpenicillin was performed on $E$. coli cells with the bacterial culture $E$. coli seeded on the meatpeptone agar. The discs were distributed on the surface of the sown agar by tweezers and then soaked in a solution of benzylpenicillin of $10 \mu 1$ per disc. The cups were then incubated in a thermostat at $37^{\circ} \mathrm{C}$ for $18 \mathrm{~h}$.

The antibacterial activity was evaluated by measuring the average diameter of the lysis zone. The measurement was carried out as follows: the cups were placed upside down on a dark matte surface so that the light fell on them at an angle of $45^{\circ}$ (accounting for the reflected light), after which the diameter of growth retardation zones was measured using calipers (the measurement error is $\pm 0.1 \mathrm{~mm}$ ). The results obtained during the experiment were checked for reliability using the Student's criterion (the level of statistical significance $<0.05$ ). The diameter of each lysis zone was measured at least 10 times, after which the average diameter was calculated. To increase the reliability of the results, independent series of repeated experiments were conducted.

The increase in antibacterial activity of benzylpenicillin sodium salt after PMF processing discovered in of this study can be explained by the transition of the PMF-processed molecule to an excited state when its conformation changes slightly. It was also found that the biological effect of the PMF action on drugs decreases with time, being maximally manifested within the day. PMF treatment of benzylpenicillin sodium was not accompanied by an increase in its acute toxicity or emergence of free radicals.

The aim of the work is to study the structural changes in a molecule of benzylpenicillin sodium salt after exposure to a pulsed magnetic field.

\section{Experimental Procedure}

The study of benzylpenicillin sodium salt was carried out at the PMF parameters (Table 1) with the frequency of $\mathrm{f}=40 \mathrm{kHz}$. The exposure time of the test drug to the PMF was $0.0002-0.005 \mathrm{~s}[12,13]$. 
Table 1 Parameters of the PMF action on benzylpenicillin sodium salt.

\begin{tabular}{llll}
$\mathrm{W}$ (energy), $\mathrm{kJ}$ & 0.45 & 2.49 & 4.11 \\
\hline $\mathrm{H}$ (intensity), $10^{6} \mathrm{~A} / \mathrm{m}$ & 0.09 & 0.50 & 0.82 \\
\hline
\end{tabular}

$\mathrm{f}$ (frequency), $\mathrm{kHz}$

40

Control samples of the drug were not exposed to the PMF. To register the spectra, the powder was thoroughly ground with $\mathrm{KBr}$ in an agate mortar at the ratio of 1:100. The test mixture was placed in a cuvette in a special holder, after which the diffuse reflection spectra were recorded.

As is well-known, the most informative regions of the Fourier spectra are the medium-frequency $\left(1000-1970 \mathrm{~cm}^{-1}\right)$ and high-frequency $\quad(3200$ $3800 \mathrm{~cm}^{-1}$ ) regions. The spectra were recorded using a FSM-2201 Fourier spectrophotometer (Infaspec, Russia) in the wavenumber range from $470 \mathrm{~cm}^{-1}$ to $5000 \mathrm{~cm}^{-1}$.
The studied spectra were recorded with a spectral resolution Fspec software (Monitoring, Russia). The limit of the permissible absolute error of the wavenumber scale on the device is $\pm 0.05 \mathrm{~cm}^{-1}$, the error of measuring the absorption intensity is $0.05 \%$.

\section{Results and Discussion}

Figs. 3 and 4 present spectra of benzylpenicillin as a result of the PMF action for two regions of the spectrum. In the region of $\mathrm{N}-\mathrm{H}$ valence oscillations, the following bands are observed: $3320 \mathrm{~cm}^{-1}, 3355 \mathrm{~cm}^{-1}, 3369 \mathrm{~cm}^{-1}$ (Fig. 3).

Table 2 presents the changes recorded in the Fourier spectrum when the antibiotic was exposed to the PMF. The intensities of the bands $3320 \mathrm{~cm}^{-1}$ and $3369 \mathrm{~cm}^{-1}$ decrease in proportion to the magnetic field intensity. The intensity of the $3355 \mathrm{~cm}^{-1}$ band first decreases slightly (by about $3 \%$ ) and then returns to its original state.

Fig. 4 presents the bands of absorption of the pharmacy antibiotic in the carbonyl region.

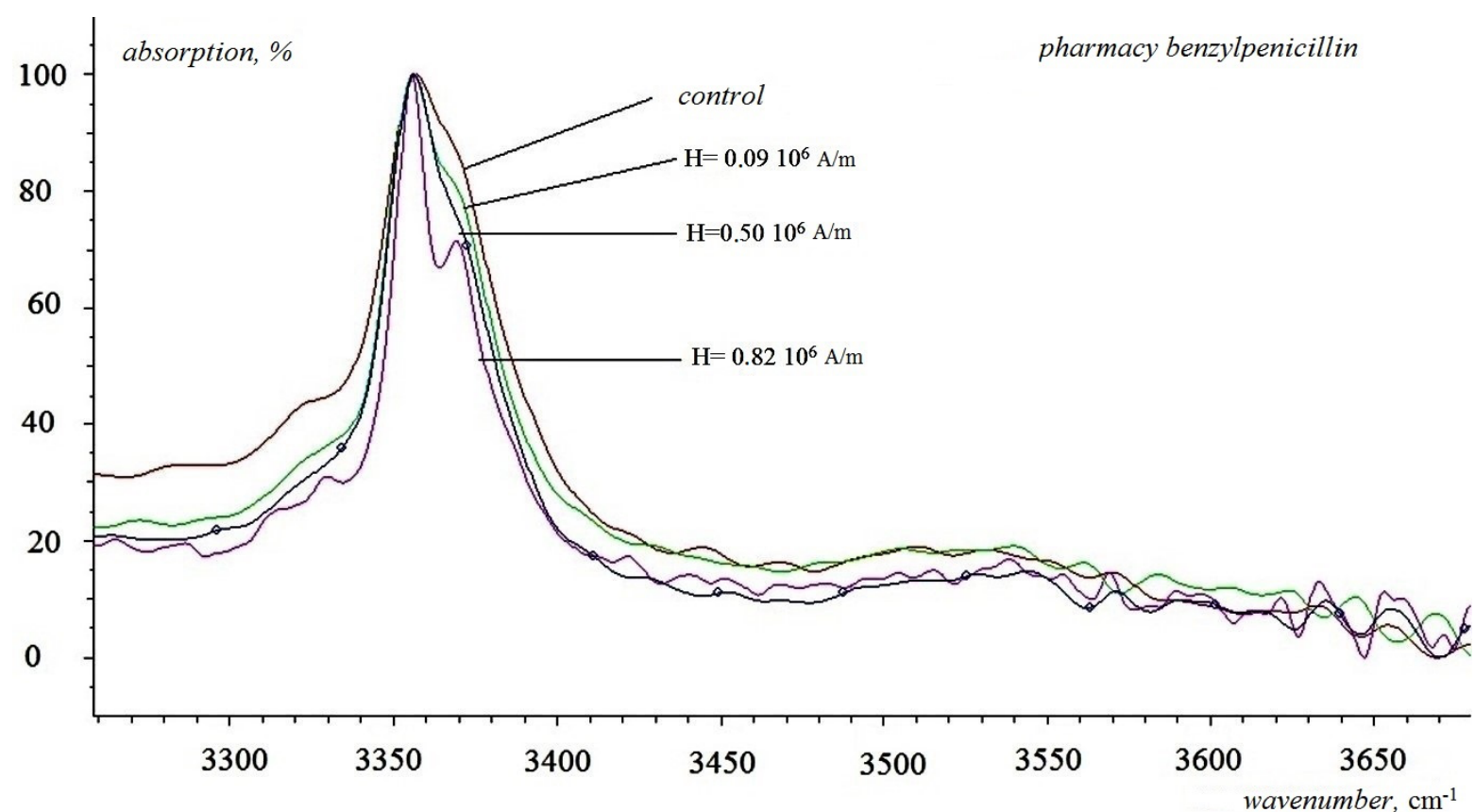

Fig. 3 Fourier spectra of the antibiotic after the PMF processing.

Table 2 Changes in the Fourier spectrum under the PMF action on benzylpenicillin.

\begin{tabular}{ccccc} 
Band, $\mathrm{cm}^{-1}$ & Control & $\begin{array}{c}\text { Processing at intensity } \\
\mathrm{H}=0.09 \times 10^{6} \mathrm{~A} / \mathrm{m}\end{array}$ & $\begin{array}{c}\text { Processing at intensity } \\
\mathrm{H}=0.50 \times 10^{6} \mathrm{~A} / \mathrm{m}\end{array}$ & $\begin{array}{c}\text { Processing at intensity } \\
\mathrm{H}=0.82 \times 10^{6} \mathrm{~A} / \mathrm{m}\end{array}$ \\
\hline 3355 & 100 & 96.7 & 96.7 & 100 \\
\hline 3369 & 85.6 & 80.6 & 73.8 & 70.4 \\
\hline 3320 & 42.6 & 33.5 & 29.6 & 27
\end{tabular}




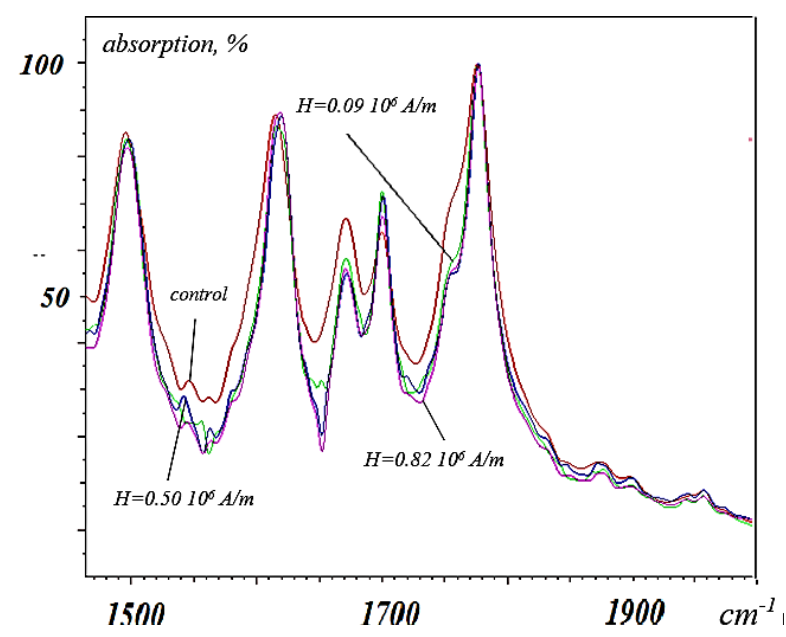

Fig. 4 Absorption bands in the carbonyl region of the Fourier spectrum of pharmacy antibiotic.

The $1700 \mathrm{~cm}^{-1}$ region displays the bands that correspond to valence oscillations $\mathrm{C}=\mathrm{O}$ of the peptide group $\left(1698 \mathrm{~cm}^{-1}\right)$, oscillations $\mathrm{C}=\mathrm{O}$ of the group attached to the $\beta$-lactam ring (the $1773 \mathrm{~cm}^{-1}$ band), the $1672 \mathrm{~cm}^{-1}$ band belongs to the complex oscillation of the amide group.

The following conclusions can be drawn from the given spectra:

1. Intensity of the $1773 \mathrm{~cm}^{-1}$ band does not depend on the PMF processing of the drug.

2. Intensities of the $1672 \mathrm{~cm}^{-1}$ and the $1699 \mathrm{~cm}^{-1}$ are redistributed depending on the power of the PMF action: the intensity of the high-frequency band increases, and the intensity of the low-frequency band decreases.

In our previous work [14], we found that the enhancement of the biological effect from the PMF action on the antibiotic disappears after about 24 hours. The Fourier spectra were studied for the effect of the repeated exposure to the PMF.

The spectra of the powdered benzylpenicillin in potassium bromide were studied under the following experimental conditions:

- sample 1 was exposed to the PMF once, i.e. the spectra were recorded after 24 hours;

- sample 2 was exposed to the PMF twice with a $24 \mathrm{~h}$ interval, i.e. the spectra were recorded after $48 \mathrm{~h}$;

- sample 3 was exposed to the PMF three times with an interval of $24 \mathrm{~h}$, i.e. the spectra were recorded after $72 \mathrm{~h}$.

When processing the powdered antibiotic by the pulsed magnetic field with the intensity $\mathrm{H}=0.09 \times 10^{6} \mathrm{~A} / \mathrm{m}$, it was noticed that in the region of $\mathrm{N}-\mathrm{H}$ valence oscillations, the band intensity in the $3400 \mathrm{~cm}^{-1}$ region decreases 24 and $48 \mathrm{~h}$ after the PMF action on the same drug compared to the unprocessed drug, and the maxima become more distinct. In this case, the band of $3470 \mathrm{~cm}^{-1}$ decreases in intensity more substantially at PMF intensities $\mathrm{H}=0.09 \times 10^{6} \mathrm{~A} / \mathrm{m}$ and $\mathrm{H}=0.50 \times 10^{6} \mathrm{~A} / \mathrm{m}$. Apparently, there is a break in the hydrogen bonds with the water molecules contained in $\mathrm{KBr}$. In the low-frequency region of the spectrum, no changes are observed. At intensity $\mathrm{H}=0.82 \times 10^{6} \mathrm{~A} / \mathrm{m}$, a decrease in intensity of bands of $3335 \mathrm{~cm}^{-1}$ and $3370 \mathrm{~cm}^{-1}$ was observed in the region of oscillations of the carbonyl group $[15,16]$.

When the drug was re-processed with the PMF on the second day, a change in the intensity of the bands of the Fourier spectra was observed, and when the PMF was applied to the antibiotic twice for a period less than 48 hours, the band intensity also decreased. Thus it can be assumed that the repeated PMF treatment of the same drug has an effect on the intensity of oscillations. For example, when comparing the $3335 \mathrm{~cm}^{-1}$ band without the PMF action to a two-time exposure, the intensity decreases by about $13 \%$. It is assumed that after the highintensity PMF action, the charge accumulates in the drug, and after the elimination of the magnetic field, the effect gradually disappears since, as mentioned above, the time of retention of the PMF effect is about $24 \mathrm{~h}$.

According to the results obtained, the changes in the spectra of benzylpenicillin sodium salt suggest that the hydrogen bonds play a decisive role in the change in biological activity. The recorded changes in the functional groupings of the antibiotic may be due to the fact that when the drug molecule is placed in the pulsed magnetic field, the orientation of the molecule changes relative to the direction of the applied PMF.

It is well-known that penicillins cause the death of microorganisms by blocking the inclusion of acetylmuram peptides in the growing cell walls of bacteria acting mainly on the enzyme D-D-transpeptidase, which cleaves the terminal D-alanine from $\mathrm{N}$-phcetilmuramylpentapeptide and catalyzes the formation of cross-links. Therefore, the antibacterial activity of $\beta$-lactam antibiotics is primarily associated with the suppression of the activity of transpeptidase. D-alanyl-D-alanine plays the role of an identification mark for the enzyme. Another factor is acylation of transpeptidase by peptidoglycan, accompanied by the loss of one D-alanine molecule:

Enzyme + R-D-Ala-D-Ala $\rightarrow$ R-D-Ala-enzyme + D-Ala

Then transpeptidase is regenerated binding the terminal residue of $\mathrm{D}$-alanine of the peptidoglycan molecule to a chain of four glycine molecules (connected to the lysine residue), the second molecule of peptidoglycan. Penicillins having groupings similar to D-alanyl-D-Alanine in the region of the $\beta$-lactam ring (Fig. 5) irreversibly inhibit transpeptidase, which causes the death of $E$. coli. cells.

Therefore, changes in the valence angles of the amide grouping near the $\beta$-lactam ring after exposure to the pulsed magnetic field can cause minor changes in the structural conformation of the benzylpenicillin molecule and contribute to an increase in the affinity to the active centre of the enzyme, thereby increasing the antibacterial activity of the drug. 


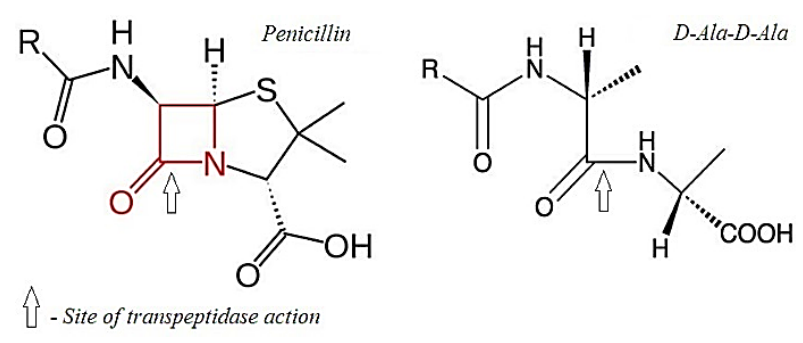

Fig. 5 Comparison of the molecular structure of penicillin and D-alanyl-D-alanine.

\section{Conclusion}

An increase in power of the magnetic field action on benzylpenicillin sodium salt leads to a decrease in the intensity of the $3320 \mathrm{~cm}^{-1}$ and the $3369 \mathrm{~cm}^{-1}$ bands. Changes in the amide group were recorded depending on the application of the PMF to the antibiotic. Perhaps, the changes are produced by the geometry of the molecule.
Changes in the structural conformation of the benzylpenicillin sodium salt molecule, processed by the pulsed magnetic field, could contribute to an increase in the affinity to the active centre of the enzyme of transpeptidase, thereby possibly increasing the antibacterial activity of the drug. The observed changes in the spectra of benzylpenicillin sodium salt after PMF processing are small, so further studies are required to study the PMF action on the structure of the antibiotic and its antibacterial activity.

\section{Disclosures}

All authors declare that there is no conflict of interests in this paper.

\section{Acknowledgements}

This research was supported by the UMNIK program of the Innovation Promotion Fund.

\section{References}

1. B. Olivares, F. Martínez, L. Rivas, C. Calderón, J. M. Munita, and P. R. Campodonico, "A natural deep eutectic solvent formulated to stabilize $\beta$-lactam antibiotics," Scientific Reports 8(1), 14900 (2018).

2. A. I. Varakin, Y. V. Seryanov, and N. V. Arkhipova, "Quantum-chemical modeling of antibiotic molecules and anions and the nature of their transport in biological membranes," Bashkir Chemical Journal 16(3), 116-121 (2009) [in Russian].

3. T. A. Krysanova, D. L. Kotova, and E.G. Davydova, "Interactions in the system water-solid samples of sodium salt of benzylpenicillin,” Bulletin of Voronezh State University. Series: Chemistry, Biology, Pharmacy 2, 37-41 (2012) [in Russian].

4. J. Potticary, L. R. Terry, C. Bell, A. N. Papanikolopoulos, P. C. M. Christianen, H. Engelkamp, A. M. Collins, C. Fontanesi, G. Kociok-Köhn, S. Crampin, E. D. Como, and S. R. Hall, "An unforeseen polymorph of coronene by the application of magnetic fields during crystal growth," Nature Communications 7(1), 11555 (2016).

5. J. G. Brandenburg, J. Potticary, H. A. Sparkes, S. L. Price, and S. R. Hall, "Thermal Expansion of Carbamazepine: Systematic Crystallographic Measurements Challenge Quantum Chemical Calculations," The Journal of Physical Chemistry Letters 8 (17), 4319-4324 (2017).

6. V. Shaporov, "The effect of magnetic field on the action of medicinal substances," Medchitalka (accessed 17 March 2021). [https://www.medchitalka.ru/medicina_segodnya/medicinskaya_orbita/3398.html] [in Russian].

7. N. L. Shimanovsky, M. A. Epinetov, and M. Y. Melnikov, Molecular and Nanopharmacology, Fizmatlit, Moscow, (2009) [in Russian]. ISBN 978-5-9221-1208-6.

8. V.A. Glushchenkov, T. I. Vasilyeva, P. P. Purigin, I. A. Belyaeva, N. A. Rodenko, A. K. Madyarova, and R. Ju. Jusupov, "Changes in the Antibacterial Activity of Benzylpenicillin Exposed to a Pulsed High-Intensity Magnetic Field," Biophysics 64 (2), 214-223 (2019).

9. L. T. Musina, Physiology of bacteria: Instructional guidelines for teachers and students of medical universities, KSMU, Kazan, Russia (2001) [in Russian].

10. A. S. Labinskaya, Guide to Medical Microbiology. Private medical microbiology and etiological diagnosis of infections: Manual for postgraduate professional education of Physicians, Binom, Moscow (2010) [in Russian].

11. N. A. Klenova, Laboratory Workshop on Microbiology, Samara University, Samara (2012) [in Russian].

12. V. A. Glushchenkov, V. F. Karpukhin, Magnetic-Impulse Materials Processing Technlogy, Fedorov, Samara, Russia (2014) [in Russian].

13. R. Yu. Yusupov, V.A. Glushchenkov, Power Units for Magnetic-Impulse Materials Processing, Fedorov, Samara, Russia (2013) [in Russian].

14. N. A. Rodenko, T. I. Vasilieva, I. A. Belyaeva, V. A. Gluschenkov, P. P. Purygin, A. V. Samorodov, and L. I. Bashirova, "Research on the safety of benzylpenicillin sodium salt after exposure to a pulsed magnetic field," Butlerov Communications 58(6), 123-129 (2019).

15. Ch. N. Rao, Electronic Spectra in Chemistry, Mir, Moscow, Russia (1964) [in Russian].

16. O. V. Sverdlova, Electronic Spectra in Organic Chemistry, Chemistry, Leningrad, Russia (1973) [in Russian]. 